\title{
Extreme Dark Matter Dominated Dwarfs
}

\author{
Mark I. Wilkinson, Jan T. Kleyna, N. Wyn Evans, Gerard F. Gilmore \\ Institute of Astronomy, Madingley Road, Cambridge CB3 OHA, UK
}

\begin{abstract}
The results of a large radial velocity survey of the Draco and Ursa Minor dwarf spheroidal galaxies are presented. The velocity dispersion profiles of both objects are very similar: initially the dispersion increases with radius, while at radii approaching the outer limit of the stellar distribution we observe a sharp fall-off. We present the results of mass modeling based on these new data - the mass to light ratios are found to be about $400 \mathrm{M}_{\odot} / \mathrm{L}_{\odot}$. Evidence is also presented that the dark matter in the central regions of UMi has a core-like distribution. Finally, we demonstrate that the inner regions of UMi have not been affected by tides and discuss the implications of our data for MOdified Newtonian Dynamics (MOND).
\end{abstract}

\section{Introduction}

The Local Group dwarf spheroidal (dSph) galaxies present a unique opportunity for the study of dark matter (DM) on small scales. The large estimated mass to light (M/L) ratios for several dSphs (e.g. Draco) and the apparent absence of DM in globular clusters make dSphs the smallest and most DM dominated stellar systems in the universe. We have an on-going program of observations to study the internal kinematics of Local Group dwarfs using individual stellar velocities. The results presented here are based on observations of Draco and Ursa Minor (UMi) with the AF2/WYFFOS multifibre spectrograph on the William Herschel Telescope, La Palma. We have concentrated on obtaining velocities for stars at large radii as these place the tightest constraints on the halo mass and extent. In each galaxy, we now have a data set of about two hundred giant stars which extends to the edge of the stellar distribution. All velocities were measured using the CaII triplet line with typical errors in the range $2-5 \mathrm{~km} \mathrm{~s}^{-1}$.

\section{New velocity dispersion profiles}

Figure 1 shows the radial variation of the line of sight velocity dispersion in Draco and UMi. As we observed previously in Draco, the velocity dispersion profile in UMi rises gently from the centre. However, the data show that at the largest radii observed the dispersion drops sharply - this is also seen in our new data set for Draco. We have analysed these new data using the twoparameter dynamical models presented in Wilkinson et al. (2002). These models incorporate a halo shape parameter $\alpha$, which allows us to consider a range of DM 

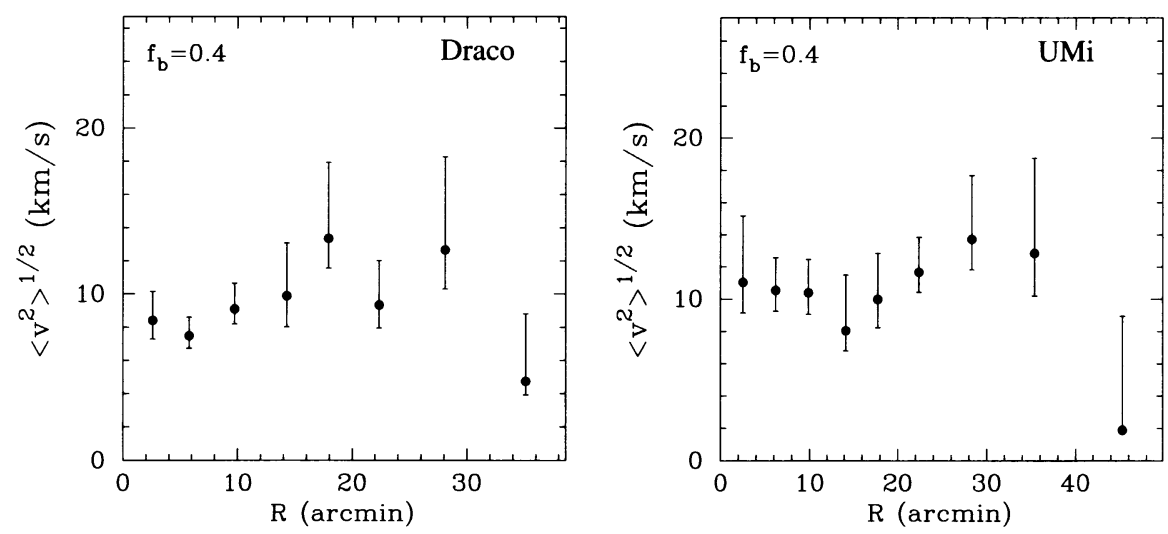

Figure 1. Radial variation of the line of sight stellar velocity dispersion in Draco and UMi. $1 \sigma$ errorbars (calculated assuming a $40 \%$ binary fraction) are shown.

profiles from mass-follows-light $(\alpha=1)$ to extended harmonic haloes $(\alpha=-2)$, and a velocity anisotropy parameter $\nu$, which is zero for an isotropic velocity distribution and positive (negative) for radial (tangential) velocity anisotropy. These models enable us to break the principal degeneracy of the problem, namely that a large observed velocity dispersion in the outer parts of a dSph can be due either to the presence of significant DM at large radii or a tangentially anisotropic velocity distribution. In the Draco $\mathrm{dSph}$ we were able to rule out the traditional mass-follows-light models at the $2.5 \sigma$ confidence level (Kleyna et al. 2001).

Figure 2 presents likelihood contours for the model parameters based on our new data sets. In the case of UMi, we have used only the data within $35^{\prime}$ as this is the region within which we are confident that tides are unimportant (see below). The best-fit model for Draco has a mass interior to 3 core radii of $8 \times 10^{7} \mathrm{M}_{\odot}$ which implies a $\mathrm{M} / \mathrm{L}$ of $440 \mathrm{M}_{\odot} / \mathrm{L}_{\odot}$; for UMi the mass within $35^{\prime}$ is $1.8 \times 10^{8} \mathrm{M}_{\odot}$ which corresponds to a $\mathrm{M} / \mathrm{L}$ of $330 \mathrm{M}_{\odot} / \mathrm{L}_{\odot}$. Both galaxies appear to have roughly isotropic velocity distributions.

We are currently investigating the possible origins of the sudden drop in the projected dispersion profiles of UMi and Draco. In Draco, the absence of other evidence for tidal effects (Odenkirchen et al. 2001, Klessen et al. 2003) makes it unclear whether tides can be implicated. In the case of UMi, as we discuss below, its outer parts may be susceptible to sculpting by the Milky Way and the falling dispersion may be a result of this process. The similarity of the best-fit mass models and the observed dispersion profiles is particularly striking given the well-known morphological differences between Draco and UMi. This raises the intriguing possibility that a universal $\mathrm{dSph}$ halo profile might exist. We note that the fall-off at large radii calls into question recent claims that the dSphs must reside in haloes which extend far beyond the edge of the light distribution (e.g. Stoehr et al. 2002). It also represents a serious difficulty for tidal disruption models of dSphs (e.g. Gómez-Flechoso \& Martínez-Delgado $2003)$ as these models predict a radially rising velocity dispersion. 


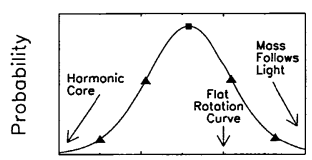

Draco

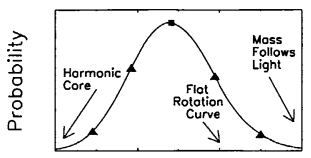

UMi
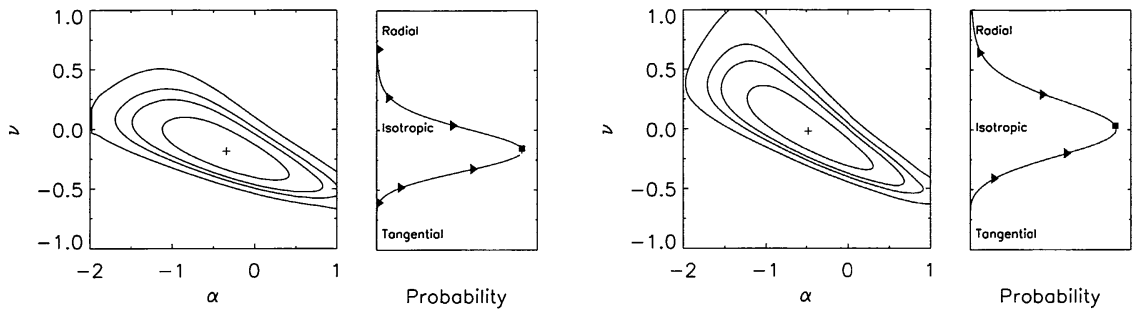

Figure 2. Likelihood contours for halo shape $\alpha$ and velocity anisotropy $\nu$ using data from Draco(left) and UMi (right).

\section{The Halo of Ursa Minor}

In contrast to the featureless distribution of stars in Draco, UMi exhibits substantial morphological distortions (Fig. 3): UMi is noticeably elongated $(\epsilon \sim 0.6)$ and there is a secondary clump of stars offset from the galaxy centre. It has been argued that this feature cannot be long-lived as substructure would be erased on a timescale of a few crossing times $(\sim 200 \mathrm{Myr})$. This has fuelled speculation that UMi is in the process of being tidally disrupted by the Milky Way and that the substructure is a signature of this process - however, to date no fully consistent model to explain this feature has been proposed.

Our analysis of the stellar velocity data revealed that the clump of stars is also apparent in the stellar kinematics. In the vicinity of the clump, a twocomponent velocity distribution which consists of one population whose velocity dispersion matches that of the main body of UMi $\left(8.8 \mathrm{~km} \mathrm{~s}^{-1}\right)$ and one which has a dispersion of $0.5 \mathrm{~km} \mathrm{~s}^{-1}$ is a significantly better fit to the data than a single component model. The large points in Figure 3 show the regions where this twocomponent model is at least 1000 times more likely than the single component model - the probability of such a situation arising by chance is less than 0.005 .

We have performed a series of $N$-body simulations to study the survival of a cold clump in a DM halo (Kleyna et al. 2003). We find that the survival of the clump for a significant fraction of a Hubble time is only possible if the halo has a central core. A cusped halo causes the rapid dispersal of the stellar clump. We conclude that if the observed substructure lies within the main body of UMi, the surrounding dark halo must have a large harmonic core. The only way to avoid this conclusion is to suppose that the cold clump forms part of a tidal tail and is merely projected onto the central regions of UMi. We are currently investigating this possibility by means of $N$-body simulations.

Palma et al. (2003) note that the light distribution in UMi is asymmetric about a North-South axis and they observe a break in the Eastern luminosity profile at about $35^{\prime}$ from the centre. As Figure 1 shows, the velocity dispersion profile also drops outside $35^{\prime}$. We now discuss whether these two observations could be due to tidal disturbance by the Milky Way. 

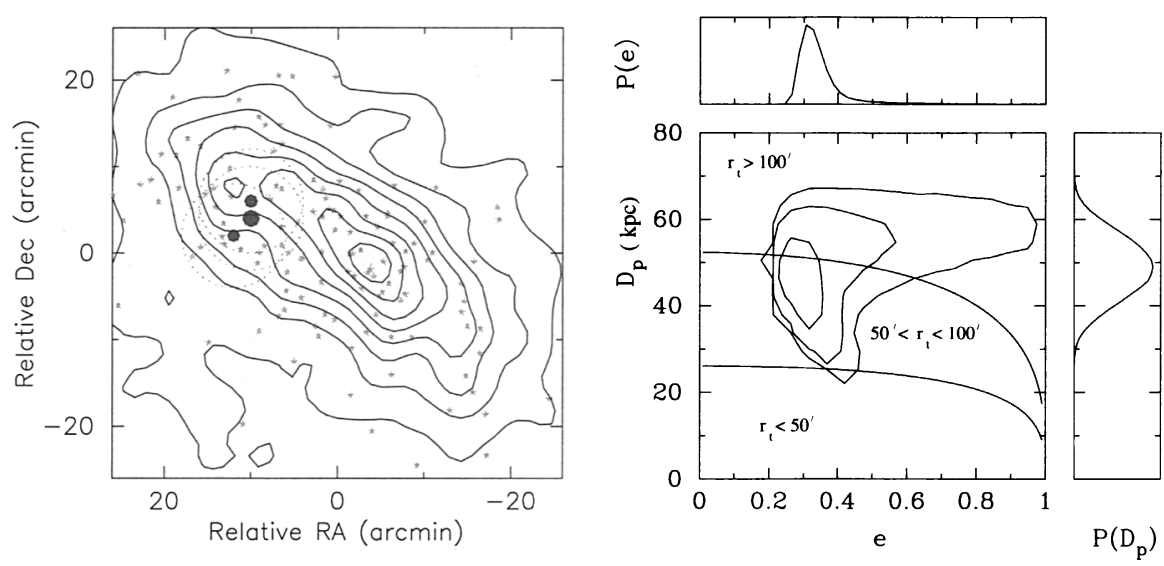

Figure 3. Left: Contour plot of UMi's stellar number density, with the secondary peak visible at the upper left. The large dots indicate the regions where a kinematically cold population has to be included to match the velocity data. Right: Likelihood contours $(1 \sigma, 2 \sigma$ and $3 \sigma)$ for the perigalactic distance $D_{\mathrm{p}}$ and eccentricity $e$ of UMi's orbit, with individual probabilities shown in the top and right panels. Overplotted are the boundaries of regions in which the perigalactic tidal radius $r_{\mathrm{t}}$ of UMi satisfies $r_{\mathrm{t}}<50^{\prime}, 50^{\prime}<r_{\mathrm{t}}<100^{\prime}$ and $r_{\mathrm{t}}>100^{\prime}$.

The mass estimate for UMi presented above was based on the velocity data from inside $35^{\prime}$. If we conservatively assume that the mass out to this radius corresponds to the total mass of $\mathrm{UMi}$, we can calculate the tidal radius of such a halo on a UMi-like orbit about the Milky Way. The likelihood contours in Figure 3 show the distribution of perigalactic distances and eccentricities for orbits which are compatible with UMi's present position, radial velocity and the recent proper motion estimate of Cudworth (priv. comm.). The plot is divided into three regions based on the associated values of the perigalactic tidal radius of UMi. As the plot clearly shows, if UMi is moving on any of the most likely orbits which correspond to its presently observed space motion, then its estimated mass within $35^{\prime}$ is sufficient to give a perigalactic tidal radius of at least $50^{\prime}$. The tidal radius at its current location will be larger because (i) its present distance is larger than its perigalactic distance and (ii) we have assumed a lower limit to the mass of $\mathrm{UMi}$, which is unlikely to be sharply truncated at $35^{\prime}$.

The results shown in Figure 3 have a number of implications for the interpretation of the UMi velocity data. First, given that its perigalactic tidal radius is likely to be larger than $50^{\prime}$, the break in the light profile seen at $35^{\prime}$ is unlikely to correspond to the transition between bound and unbound stars. Johnston et al. (2002) present the results of $N$-body simulations of the changes in the light profile of a dwarf galaxy as it orbits the Milky Way. Their simulations often display two transition radii: an outer, tidal radius $r_{\mathrm{t}}$ outside which stars are unbound and an intermediate radius $r_{\text {break }}$ where the light profile changes slope. For satellites on eccentric orbits, stars at radii between $r_{\text {break }}$ and $r_{\mathrm{t}}$ were 


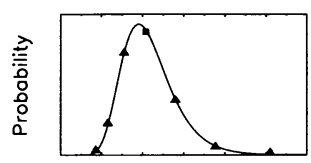

Draco

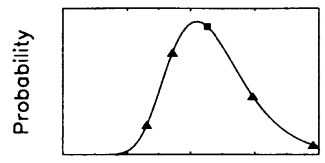

UMi
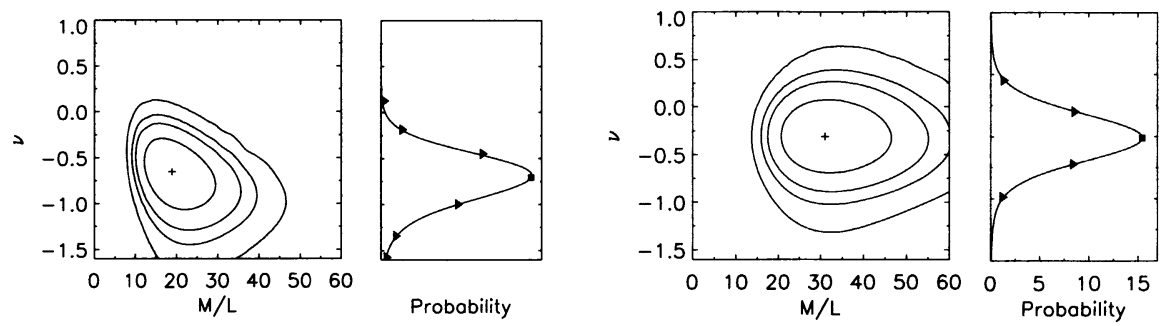

Figure 4. Likelihood contours for the stellar $\mathrm{M} / \mathrm{L}$ and velocity anisotropy $\nu$ based on a MOND analysis of the velocity data.

heated during previous close approaches to the parent galaxy but have not yet become unbound. This provides a natural explanation for the features observed in the light distribution of UMi between $35^{\prime}$ and $50^{\prime}$ - in this picture, the break radius would be at $35^{\prime}$ and any truncation would occur at the tidal radius, somewhat outside $50^{\prime}$. In this picture, none of the irregularities observed in the outer light profile of UMi is necessarily associated with an unbound tidal tail. We are currently investigating whether this scenario can also account for the fall-off in the velocity dispersion at large radii.

Finally, we note that another recent estimate for the proper motion of UMi places it on a strongly radial orbit with a perigalacticon of about $6 \mathrm{kpc}$ (Piatek 2003). Interestingly, the perigalactic tidal radius of UMi, if it moved on such an orbit, would be at about $35^{\prime}$. In this case, its present tidal radius would still lie beyond $50^{\prime}$ and our conclusions would be unchanged.

\section{MOND and Draco/UMi}

MOdified Newtonian Dynamics (MOND) has been proposed as an alternative to DM which explains the flat rotation curves of spiral galaxies in terms of a modification of Newton's law of gravitation at low accelerations (e.g. Milgrom 1983). The dSphs are interesting systems in which to test this picture because their internal kinematics suggest the presence of very large amounts of DM. We have analysed our velocity data for both Draco and UMi using a two-parameter MOND model in which the M/L of the stellar population and the anisotropy $\nu$ of its velocity distribution are free parameters. We represent the stellar distribution (which in this model provides all the gravitating mass) by a Plummer sphere and assume that the MOND acceleration constant is $a_{0}=2 \times 10^{-8} \mathrm{~cm} \mathrm{~s}^{-2}$. The contour plots in Figure 4 summarise the results of this analysis. In both galaxies the data suggest mild tangential velocity anisotropy although, for UMi, an isotropic distribution is within the $1 \sigma$ contour. Of greater significance, however, is the estimate for the stellar M/L. For Draco and UMi the most likely $\mathrm{M} / \mathrm{L}$ values are $19 \mathrm{M}_{\odot} / \mathrm{L}_{\odot}$ and $32 \mathrm{M}_{\odot} / \mathrm{L}_{\odot}$, respectively. A comparison of the stellar 
populations of UMi and Draco with those of globular clusters suggests that the $\mathrm{M} / \mathrm{L}$ should lie in the range $1-4.3 \mathrm{M}_{\odot} / \mathrm{L}_{\odot}$. Our MOND estimate disagrees with this value by more than $3 \sigma$, and implies either a very unusual stellar population or the existence of an unseen component in these galaxies.

While these results appear to be uncomfortable for MOND, we note that we have made a number of simplifying assumptions in our modeling. First, we have carried out the MOND analysis in the limit that both Draco and UMi are isolated systems. Second, we have used a spherical model for the stellar distribution of both galaxies. Although neither of these assumptions is completely valid in the case of UMi, only the outer $10 \%$ of the stars in our Draco data set lie in the non-isolated regime. Further, replacing the stellar distribution of Draco by an appropriately flattened model would reduce the M/L by at most $20 \%$.

\section{Conclusions and Future observations}

We have demonstrated that both the Draco and UMi dSphs contain large amounts of DM. However, a complete understanding of these dSphs is still lacking and the origin of the fall-off in their velocity dispersions at large radii remains unclear. Our forthcoming VLT observations of the distant Leo I and Leo II dwarfs will establish whether such a fall-off is a generic feature of dSph profiles or is particular to the nearby objects and hence will clarify the extent to which tides may be involved.

\section{References}

Gómez-Flechoso, M. Á. \& Martínez-Delgado, D. 2003, ApJ, 586, L123

Johnston, K. V., Choi, P. I., Guhathakurta, P. 2002, AJ, 124, 127

Klessen, R. S., Grebel E. K., Harbeck D., 2003, ApJ, 589, 798

Kleyna, J. T., Wilkinson, M. I., Evans, N. W., \& Gilmore, G. 2001, ApJ, 563, L115

Kleyna, J. T., Wilkinson, M. I., Gilmore, G., \& Evans, N. W. 2003, ApJ, 588, L21

Milgrom M., 1983, ApJ, 270, 365

Odenkirchen, M. et al. 2001, AJ, 122, 2538

Palma, C., Majewski, S. R., Siegel, M. H., Patterson, R. J., Ostheimer, J. C. \& Link, R., 2003, AJ, 125, 1352

Piatek, S. 2003, in Satellites \& Tidal Streams, ASP Conf. Ser., in press

Stoehr, F., White, S., Tormen, G. \& Springel, V. 2002, MNRAS, 335, L84

Wilkinson, M. I., Kleyna, J. T., Evans, N. W., \& Gilmore, G. 2002, MNRAS, 330,778 